\title{
Guidance Navigation and Control for Chang'E-5 Powered Descent
}

\author{
Honghua Zhang, ${ }^{1,2}$ Ji Li $\mathbb{D}^{1,2}$ Zeguo Wang $\mathbb{D}^{1},{ }^{1}$ and Yifeng Guan ${ }^{1,2}$ \\ ${ }^{1}$ Beijing Institute of Control Engineering, Beijing 100190, China \\ ${ }^{2}$ Science and Technology on Space Intelligent Control Laboratory, Beijing 100190, China
}

Correspondence should be addressed to Zeguo Wang; wang.zeguo@qq.com

Received 2 April 2021; Accepted 24 June 2021; Published 13 July 2021

Copyright @ 2021 Honghua Zhang et al. Exclusive Licensee Beijing Institute of Technology Press. Distributed under a Creative Commons Attribution License (CC BY 4.0).

\begin{abstract}
To achieve the goal of collecting lunar samples and return to the Earth for the Chang'E-5 spacecraft, the lander and ascender module (LAM) of the Chang'E-5 spacecraft successfully landed on the lunar surface on 1 Dec., 2020. The guidance, navigation, and control (GNC) system is one of the critical systems to perform this task. The GNC system of previous missions, Chang'E-3 and Chang'E-4, provides the baseline design for the Chang'E-5 LAM, and the new characteristics of the LAM, like larger mass and liquid sloshing, also bring new challenges for the GNC design. The GNC design for the descent and landing is presented in this paper. The guidance methods implemented in the powered descent are presented in detail for each phase. Propellant consumption and hazard avoidance should be particularly considered in the design. A reconfigurable attitude control is adopted which consists of the quaternion partition control, phase and gain stabilization filter, and dual observer. This controller could provide fast attitude maneuver and better system robustness. For the navigation, an intelligent heterogeneous sensor data fusion method is presented, and it is applied for the inertial measurement unit and velocimeter data. Finally, the flight results of the LAM are shown. Navigation sensors were able to provide valid measurement data during descent, and the thrusters and the main engine operated well as expected. Therefore, a successful soft lunar landing was achieved by the LAM.
\end{abstract}

\section{Introduction}

According to the Chang'E project plan [1], the mission objective of the Chang'E-5 spacecraft is to collect lunar surface samples and return to the Earth. To achieve this mission, the Chang'E-5 spacecraft consists of four vehicles: the orbiter, lander, ascender, and returner. These vehicles were launched as a combined module from the Earth, and the lander and ascender module (LAM) separated from the orbiter and returner module (ORM) in lunar orbit. Then, the LAM landed on the surface of the moon. After collecting lunar surface samples, the ascender with samples separated from the lander on the lunar surface before it took off and injected into the lunar orbit to rendezvous and dock with the ORM. The ORM took samples from the ascender and then separated from the ascender and returned to the Earth. When the ORM went near to the Earth, the returner separated from the ORM. The returner alone carrying samples reentered into the Earth atmosphere and landed on the designated site. In this way, the mission of Chang'E-5 is completed.

In the actual flight profile, the Chang'E-5 spacecraft was launched on 24 Nov., $202004: 30: 25$ (BJT) at Wenchang.
The LAM was separated from the ORM on 30 Nov., 2020 $04: 40: 12(\mathrm{BJT})$ at about $100 \mathrm{~km} \times 100 \mathrm{~km}$ lunar orbit. After two orbit maneuvers, the LAM began powered descent on 1 Dec., $202022: 57: 18$ (BJT) at the altitude about $18 \mathrm{~km}$ and successfully landed on the target lunar surface site.

The GNC design is always a challenging task for various kinds of spacecraft with the descent and landing mission [2-4]. In previous Chang'E landing missions, Chang'E-3 and Chang'E-4 [5, 6], landing guidance navigation and control (GNC) methods have been developed and successfully applied. In Chang'E-5 LAM, the GNC system has modified some algorithms and new components are involved to improve reliability and reduce weight. Accordingly, a light surface tension tank was selected for the lander of Chang'E-5, so liquid sloshing has to be considered to meet control stability requirements during descent. In addition, to further reduce system weight, the landing and ascent functions of the GNC system are designed in sharing some sensors, attitude thrusters, and computing resources. These differences bring new challenges for the Chang'E-5 GNC design.

This paper gives a brief introduction to the key GNC design of the Chang'E-5 LAM during descent and landing 


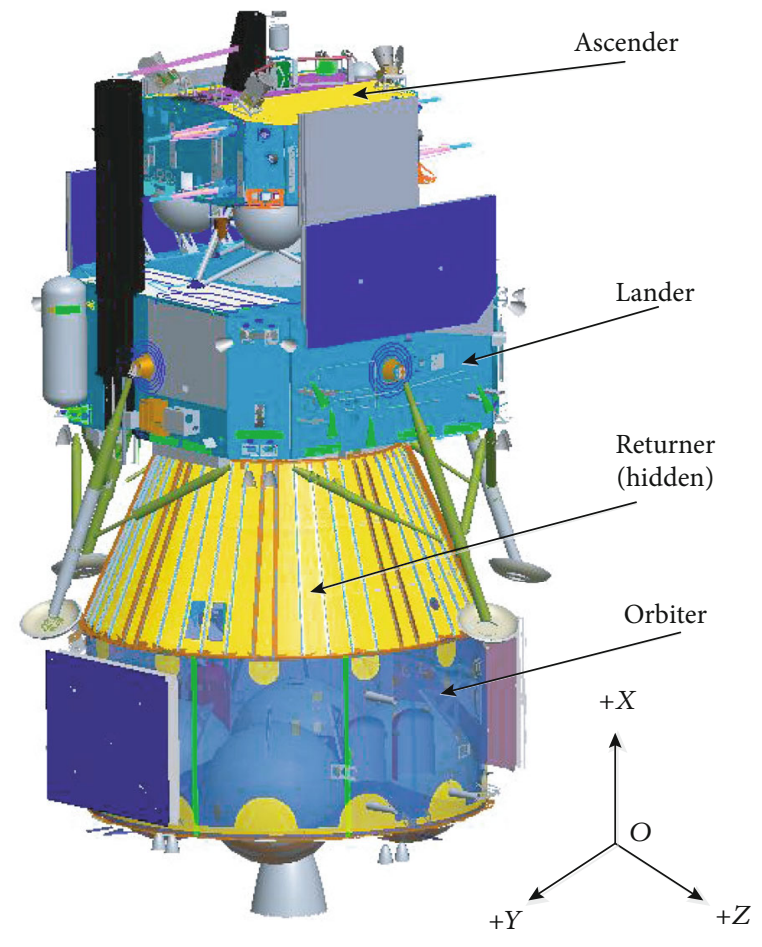

Figure 1: Chang'E-5 spacecraft configuration [7].

phase. Each flight phase guidance method is presented. The attitude controller design with consideration of propellant sloshing and analysis is also given. The descent navigation is also a challenging task, and heterogeneous sensor data fusion is implemented to improve reliability. Finally, the flight results of the LAM are presented.

\section{System Components}

The configuration of the Chang'E-5 spacecraft is shown in Figure 1. To reduce system weight, some of the landing functions are performed via components located on the ascender, like GNC computer, star trackers, sun sensors, inertial measurement units (IMU), and some attitude thrusters.

Some key system components operated in the powered descent and landing of Chang'E-5 are listed in Table 1. Their main functions are summarized here. During the descent phase, angular and acceleration increments are obtained from the IMU. According to strapdown inertial navigation algorithm and navigation filters, navigation results are updated with star sensor, radar and laser altimeters and velocimeters, and gamma sensor. With navigation results and guidance command, the $7500 \mathrm{~N}$ throttleable engine could provide adjustable thrust to realize soft landing. The $150 \mathrm{~N}$ thrusters are able to provide more attitude control torque during descent as well as horizontal thrust during fine hazard avoidance phase. All GNC algorithms are performed in the central control unit, which consists of three identical radiation-hardening computers working in the triplemodular redundancy way. Furthermore, safe landing sites are determined online with optical and laser radar (LiDAR) cameras in the coarse and fine hazard avoidance phase and
TABLE 1: Chang'E-5 GNC system components during descent.

\begin{tabular}{lc}
\hline Component name & Location \\
\hline Central control unit & Ascender \\
Sun sensor & Ascender \\
Star sensor & Ascender \\
IMU & Ascender \\
$10 \mathrm{~N}$ thruster & Ascender \\
Image processing unit & Lander \\
LiDAR camera & Lander \\
Optical camera & Lander \\
Radar altimeter & Lander \\
Radar velocimeter & Lander \\
Laser altimeter & Lander \\
Laser velocimeter & Lander \\
Gamma sensor & Lander \\
$150 \mathrm{~N}$ thruster & Lander \\
$7500 \mathrm{~N}$ throttleable engine & Lander \\
\hline
\end{tabular}

the computation of selecting landing sites is performed in the image processing unit.

\section{Intelligent Guidance}

The landing guidance used in Chang'E-5 LAM is basically derived from Chang'E-3 and Chang'E-4 [5, 6], which have various guidance methods in different descent and landing phases, and some slight changes are implemented to reduce hazard avoidance time.

3.1. Descent Trajectory. The descent trajectory planning of Chang'E-5 LAM is derived from Chang'E-3 lander, which consists of braking, pitch-up, approach, hovering, hazard avoidance, and slow-rate descent phase. The scheme of the trajectory is shown in Figure 2. The braking phase starts with the ignition of the $7500 \mathrm{~N}$ throttleable engine, and the altitude of LAM is about $18 \mathrm{~km}$. The main objective of this phase is to reduce the LAM velocity and guide LAM to the entry point of the approach phase with optimal fuel consumption. Navigation altitude correction begins at about $12 \mathrm{~km}$ with radar and laser altimeter. The pitch-up phase starts at about $2.5 \mathrm{~km}$. The attitude of LAM is reoriented quickly during this phase, and the thrust of the throttleable engine is adjusted according to program as well. The approach phase starts when the altitude reaches about $2 \mathrm{~km}$. Then, an almost constant 45-degree slant angle trajectory is followed until the altitude reaches $100 \mathrm{~m}$. Coarse hazard avoidance is conducted during this phase with optical camera. In the hovering phase, the LAM maintains the altitude as $100 \mathrm{~m}$ to image using LiDAR camera. Then, the hazard avoidance phase begins to maneuver to the new safe landing site until the altitude reaches $30 \mathrm{~m}$. Vertical trajectory is followed during slow-rate descent phase, and all thrusters and the main engine are shut down when the altitude reaches $2 \mathrm{~m}$. 


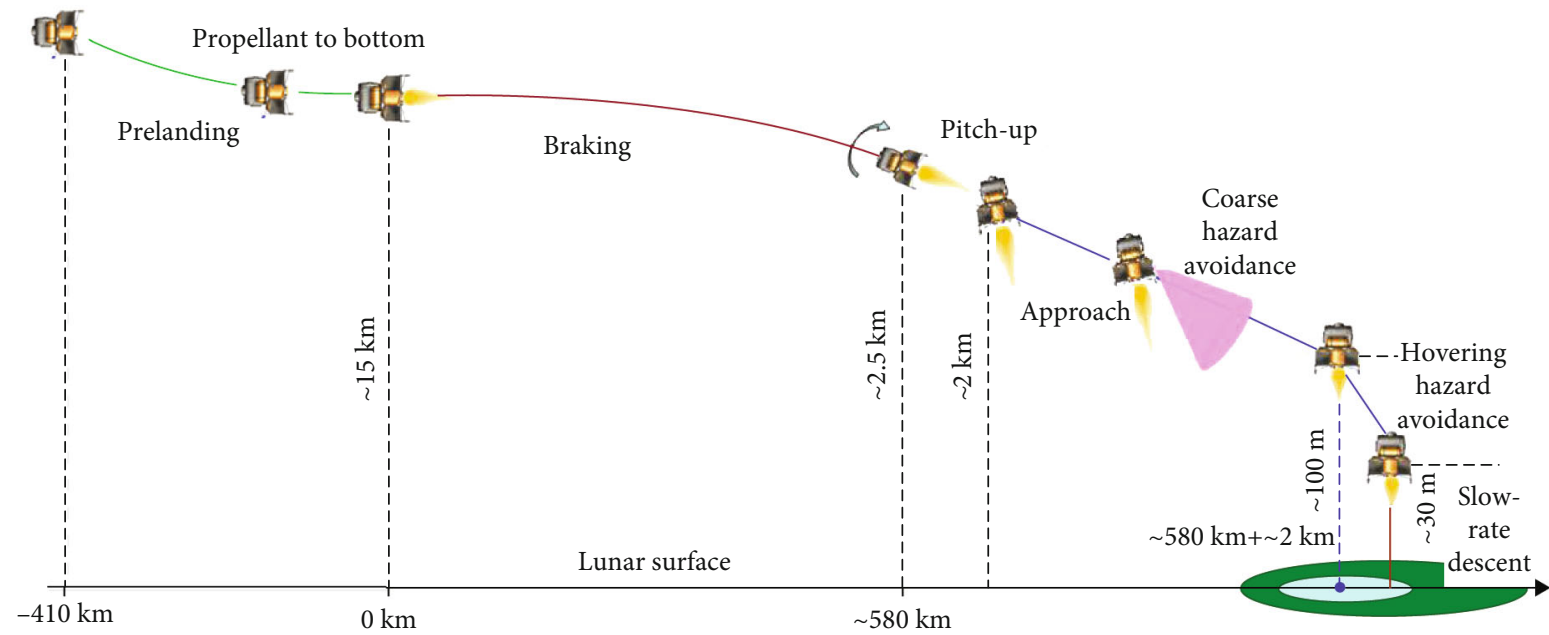

Figure 2: Scheme of Chang'E-5 LAM descent trajectory.

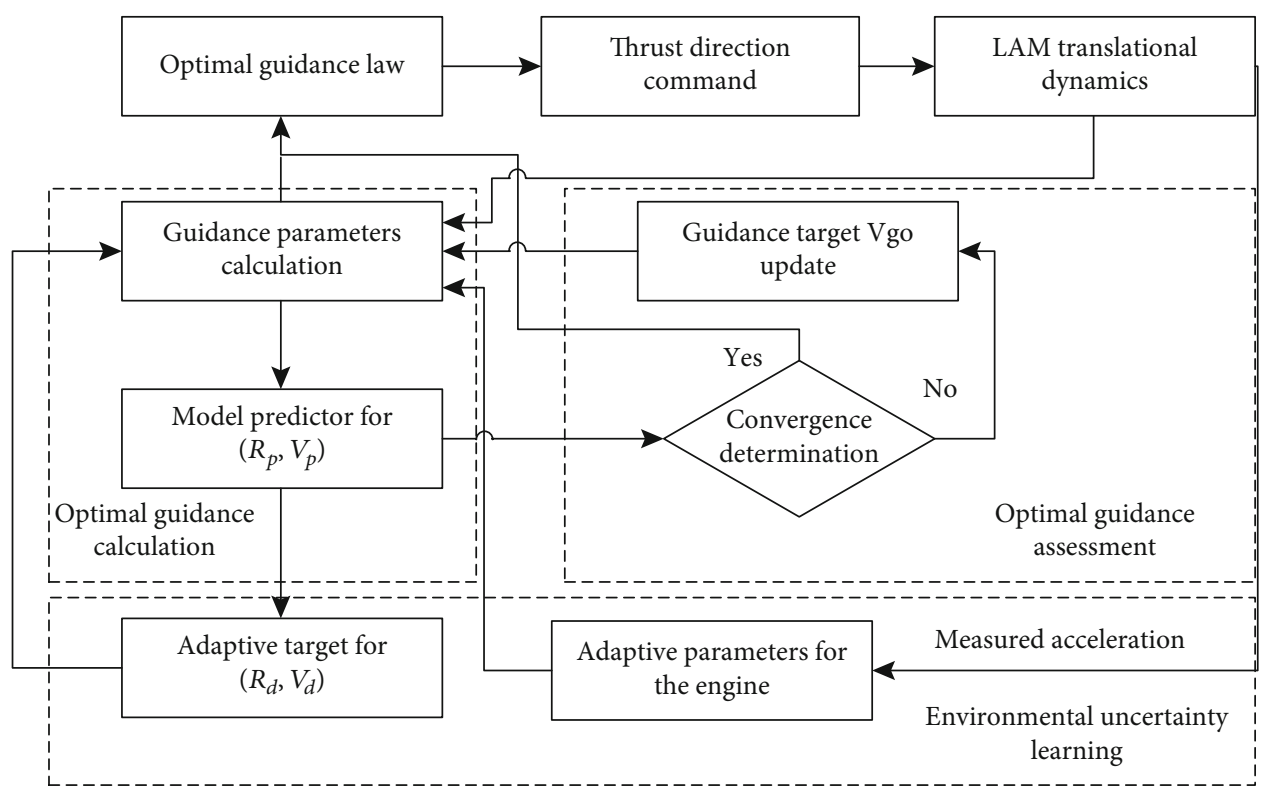

FIgure 3: Diagram of Chang'E-5 LAM intelligent guidance.

3.2. Braking Phase Guidance. During the LAM braking phase, the guidance implemented is developed from optimal control theory and the space shuttle ascent guidance method called powered explicit guidance (PEG) [8-10]. Some important advances for the PEG are made to make it applicable for the lunar descent guidance.

The diagram of this guidance method is shown in Figure 3. In general, it contains three parts: optimal guidance calculation (OGC), optimal guidance assessment (OGA), and environmental uncertainty learning (EUL). In the EUL part, the engine-specific impulse and constant maximum thrust are estimated using online acceleration measurements with the least square method. Suppose that the initial mass is $m_{0}$, the maximum thrust is $F_{\max }$, the measured acceleration is $a$, and the engine characteristic velocity is $v_{e x}$; then, the estimated coefficients are obtained as

$$
\widehat{\theta}_{F}=\left[\sum_{k=0}^{N} \phi_{F}\left(t_{k}\right) \phi_{F}^{T}\left(t_{k}\right)\right]^{-1} \sum_{k=0}^{N} \phi_{F}\left(t_{k}\right) y_{F}^{T}\left(t_{k}\right),
$$

where $\theta_{F}=\left[\tau_{F} / v_{e x}, 1 / v_{e x}\right]^{T}, \phi_{F}(t)=\left[1,-t_{k}\right]^{T}, y_{F}\left(t_{k}\right)=1 / a\left(t_{k}\right)$, $\tau_{F}=v_{e x} m_{0} / F_{\max }$, and $\widehat{\theta}_{F}$ is the estimate for $\theta_{F}$. Then, the estimated characteristic velocity is $\widehat{v}_{e x}=1 / \widehat{\theta}_{F, 2}$, and the estimated maximum thrust is $\widehat{F}_{\max }=m_{0} / \widehat{\theta}_{F, 1}$, where $\widehat{\theta}_{F, i}, \quad i=1,2$, 


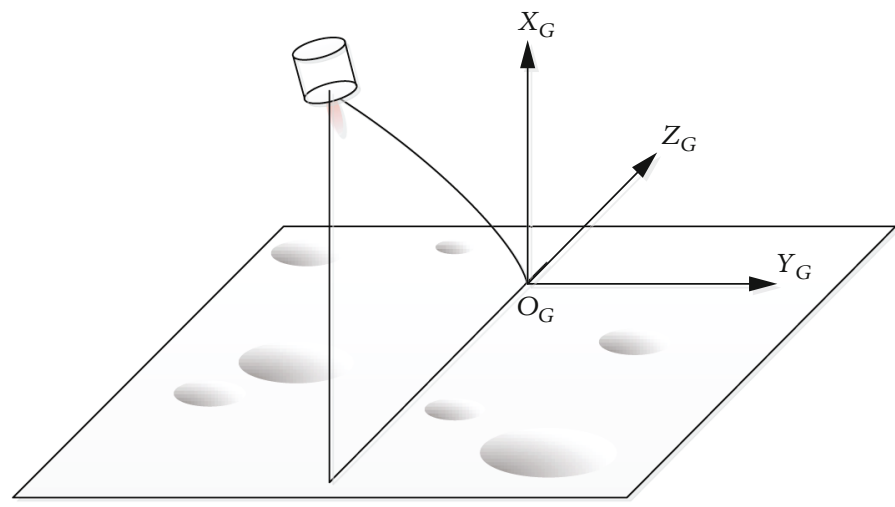

Figure 4: Chang'E-5 guidance frame during approach phase.

indicates the $i$ th element of $\widehat{\theta}_{F}$. The target terminal states are also updated online:

$$
\mathbf{R}_{d}(k)=\mathbf{R}_{d}(k-1)+C_{h}\left(r_{M}+h_{\text {If } 0}-\left\|\mathbf{R}_{\text {Ifp0 }}(k-1)\right\|\right) \frac{\mathbf{R}_{d}(k-1)}{\left\|\mathbf{R}_{d}(k-1)\right\|},
$$

$\mathbf{V}_{d}(k)=\mathbf{V}_{d}(k-1)+C_{v}\left(\mathbf{V}_{\text {If } 0}-\mathbf{V}_{\text {Ifp } 0}(k-1)\right)$,

where $\mathbf{R}_{d} \in \mathbb{R}^{3}$ and $\mathbf{V}_{d} \in \mathbb{R}^{3}$ are the target terminal states, $\mathbf{R}_{\text {Ifp } 0} \in \mathbb{R}^{3}$ and $\mathbf{V}_{\text {Ifp0 }} \in \mathbb{R}^{3}$ are predicted entry states for the approach phase, $r_{M}$ is the lunar radius, $h_{\text {If } 0}>0$ and $\mathbf{V}_{\text {If } 0} \in$ $\mathbb{R}^{3}$ are the expected nominal entry states for the approach phase, and $C_{h}$ and $C_{v}>0$ are coefficients.

In the OGA part, a target delta velocity $\mathbf{V}_{\text {go }}$ is given with the convergence assessment and model predictor. If the norm of the difference $\mathbf{V}_{\text {miss }}(k-1)=\mathbf{V}_{p}(k-1)-\mathbf{V}_{d}(k-1)$ is small enough, where $\mathbf{V}_{p}$ is the predicted terminal states for the guidance, then the convergence is obtained and $\mathbf{V}_{\mathrm{go}}$ is determined; otherwise, it should be updated as follows:

$$
\mathbf{V}_{\mathrm{go}}(k)=\mathbf{V}_{\mathrm{go}}(k-1)-\rho_{\mathrm{g}} \mathbf{V}_{\text {miss }}(k-1),
$$

where $\rho_{g}>0$ is a coefficient.

It should be noted that the output of the model predictor is the terminal state of the pitch-up phase, where a predefined open-loop guidance method is implemented, and it is also the start state of the following approach phase. In the OGC part, a linear tangent thrust parameters for the guidance is determined from $\mathbf{V}_{\text {go }}$ and adaptive parameters. With guidance parameters, the direction command of the constant thrust is determined online.

This method is an intelligent guidance method from a different point of view, since it could adapt to uncertain environment (Equations (2) and (3)) and spacecraft parameters Equation (1) using the learning-like approach. It can be interpreted as a foreground-background parallel computing method as well. The foreground calculates guidance command using previously converged parameters, and the background calculates guidance parameters using sensor measurements and adaptive guidance algorithms. The fore- ground guidance parameters are updated once the convergence is obtained by the background.

3.3. Pitch-Up Phase Guidance. The objective of the pitch-up phase is to connect the braking phase and the approach phase, from a constant thrust near fuel optimal guidance to a variable thrust polynomial guidance. The thrust direction of the LAM at the end of the braking phase is still close to horizon, while the approach phase requires an almost vertical attitude. Moreover, the constant maximum thrust is generated by the $7500 \mathrm{~N}$ engine during the braking phase, while a throttleable engine between 1500 and $5000 \mathrm{~N}$ is used during the approach phase. Therefore, a fast attitude reorientation with thrust modulation is conducted during the pitch-up phase.

3.4. Approach Phase Guidance. In the approach phase, the LAM will conduct coarse hazard avoidance and the final altitude and velocity are determined at the end of this phase. A modified polynomial guidance method [11-13] is used during this phase. The time-to-go in our method is given as follows:

$$
t_{\mathrm{go}}=\frac{\left(3 v_{t G z}+v_{G z}\right)-\sqrt{\left(3 \mathrm{v}_{t G z}+v_{G z}\right)^{2}-16 a_{t G z}\left(r_{t G z}-r_{G z}\right)}}{2 a_{t G z}},
$$

where $r_{t G z}$ and $r_{G z}$ are the target and actual terminal position along the $z$ axis in the guidance coordinate frame, as shown in Figure $4, v_{t G z}$ and $v_{G z}$ are the target and actual terminal velocity along the $z$ axis, $a_{t G z}$ is the target terminal acceleration along the $z$ axis, and $z$ axis denotes the forward direction in the trajectory plane. Then, the acceleration command could be calculated as follows:

$$
\mathbf{a}_{\mathrm{cmd}, G}=12 \frac{\mathbf{r}_{t G}-\mathbf{r}_{G}}{t_{\mathrm{go}}^{2}}-6 \frac{\mathbf{v}_{t G}+\mathbf{v}_{G}}{t_{\mathrm{go}}}+\mathbf{a}_{t G}
$$

where $\mathbf{r}_{t G}, \mathbf{v}_{t G}$, and $\mathbf{a}_{t G}$ are the target terminal position, velocity, and acceleration in the guidance coordinate frame, respectively, and $\mathbf{r}_{G}$ and $\mathbf{v}_{G}$ are the position and velocity of the LAM given by the onboard navigation system. 
The guidance parameters are determined with analytical expressions, and new guidance parameters are given once the coarse hazard avoidance algorithm provides the new safe landing site.

3.5. Hovering Phase Guidance. In the hovering phase, a scanning LiDAR camera operates to obtain the terrain map of the landing area online and a final safe landing site is determined according to the map. The LAM should maintain a constant altitude and zero velocity during this phase until the LiDAR camera completes the imaging and the new safe landing site is determined. In this phase, the vertical acceleration guidance command is designed as

$$
\mathbf{a}_{v}=\left[-c_{1}(h-100)-c_{2} \dot{h}-c_{3}\left(a_{x}-g_{m}\right)+g_{m}\right] \frac{\mathbf{r}}{\|\mathbf{r}\|},
$$

where $c_{i}, i=1,2,3$, is the guidance coefficient; $h$ is altitude of the LAM; $g_{m}$ is the lunar surface gravity acceleration constant; $\mathbf{r}$ is the position vector from the lunar center to the LAM; $a_{x}$ is the measured acceleration along the $x$ axis direction in the body frame, which should be the same as the up direction in the hovering phase. The horizontal guidance is designed the same with the hazard avoidance phase, and it will be presented in the next section.

3.6. Hazard Avoidance Phase Guidance. In the hazard avoidance phase, the LAM will maneuver to the $30 \mathrm{~m}$ altitude point above the new safe landing site obtained from the hovering phase.

The planned avoidance trajectory in vertical channel consists two parts: acceleration and deceleration, and they own the same acceleration amplitude with opposite signs. The vertical acceleration command is defined as follows:

$$
\mathbf{a}_{v}=\left[-c_{1}\left(h-h_{t}\right)-c_{2}\left(\dot{h}-\dot{h}_{t}\right)+\ddot{h}_{t}+g_{m}\right] \frac{\mathbf{r}}{\|\mathbf{r}\|},
$$

where $h_{t}$ is the reference altitude with constant acceleration. At the end of this phase, the nominal descent velocity should become $1.5 \mathrm{~m} / \mathrm{s}$, and this velocity should be maintained in the beginning of slow-rate descent phase until the altitude reaches $20 \mathrm{~m}$. Then, a constant acceleration is performed in the rest part of the slow-rate descent phase until touchdown.

For the horizontal channel, a controller with a different strategy is implemented. If the position error is larger than a threshold, then the attitude is tilted to generate horizontal thrust using the main engine. The horizontal acceleration command is defined as

$$
\mathbf{a}_{h}=-d_{1} \mathbf{R}_{I C h}-d_{2} \mathbf{V}_{I C h}-d_{3} \mathbf{a}_{I C h},
$$

where $\mathbf{R}_{I C h}, \mathbf{V}_{I C h}$, and $\mathbf{a}_{I C h}$ are the horizontal position, velocity, and acceleration relative to the target landing site; and $d_{1}$, $d_{2}$, and $d_{3}>0$ are coefficients. The final acceleration command is the sum of the vertical and the horizontal one:

$$
\mathbf{a}_{\mathrm{cmd}}=\mathbf{a}_{v}+\mathbf{a}_{h}
$$

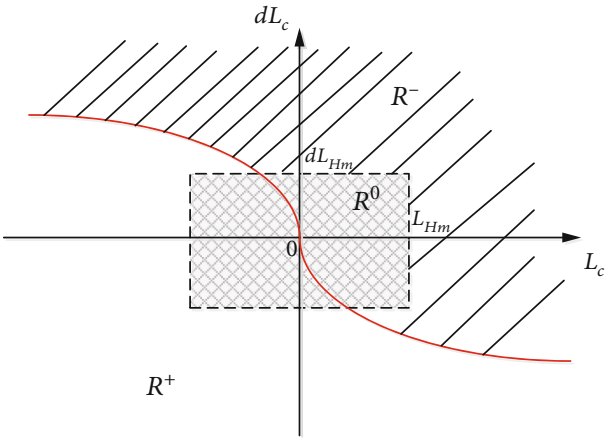

$R^{+}$: Positive thrust
$R^{-}$: Negative thrust
$R^{0}$ : PD control

FIGURE 5: Region definition of avoidance horizontal control with translational thrusters.

This acceleration command can be achieved with attitude orientation and the main engine thrust.

For the small position error, several $150 \mathrm{~N}$ thrusters are employed, which are installed in the middle of the LAM and could generate translational thrust. A bang-bang with proportional-derivative (PD) control method is used in the translational maneuver for either horizontal $y$ and $z$ axis, so that fuel sloshing is suppressed compared with simple phase-plane control method and maneuver time is reduced as well. The region definition is given in Figure 5 for either axis. The switching line of the positive and negative thrust region is given as a parabola curve, which is defined as follows:

$$
L_{c}=-\frac{d L_{c}\left|d L_{c}\right|}{a_{\mathrm{ref}}}
$$

where $L_{c}$ and $d L_{c}$ are horizontal position and velocity error to the target landing site, $F_{h}$ is the horizontal thrust magnitude, $a_{\text {ref }}=c_{s w} F_{h} / m$, and $c_{s w} \approx 1$ is the coefficient. The PD control region is defined as a box area around the origin of the phase plane to improve accuracy in small deviations, that is, $\left|L_{c}\right| \leq L_{H m}$ and $\left|d L_{c}\right| \leq d L_{H m}$, where $L_{H m}$ and $d L_{H m}$ are the box area boundary. In the PD control region, the horizontal acceleration command for either $y$ or $z$ axis is defined as follows:

$$
a_{c}=-k_{p s} L_{c}-k_{d s} d L_{c} \text {. }
$$

A pulse-width-modulation (PWM) method [14] is used to achieve the horizontal command with $150 \mathrm{~N}$ thrusters.

\section{Reconfigurable Attitude Control with Liquid Sloshing}

The diagram of the LAM reconfigurable attitude control design is shown in Figure 6. The red line is used to indicate 


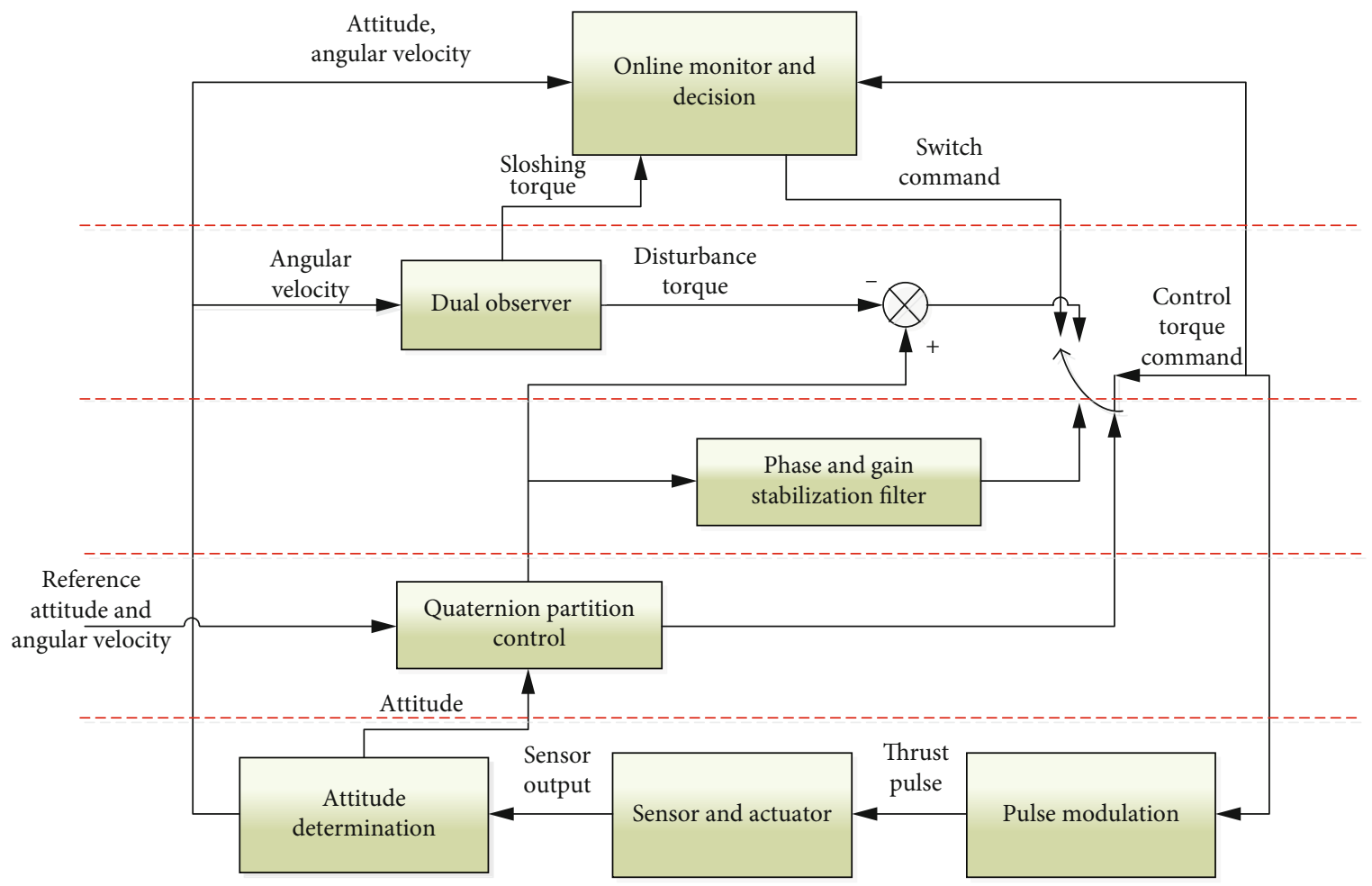

FigURE 6: Diagram of the LAM reconfigurable control.

that the reconfigurable control diagram could be divided into 5 parts. For a rigid spacecraft, the quaternion partition control alone could provide satisfying control performance [15]. However, for a liquid-filled spacecraft with sloshing effects, a reconfigurable controller is preferred, which combines several functions. Some important functions are online monitor and decision, quaternion partition control, dual observer, and phase and gain stabilization filter. The prime controller to deal with sloshing is the phase and gain stabilization filter. The controller could also be switched to a backup controller using a disturbance estimate and compensation method.

The switch decision is made by the online monitor and decision function. It takes attitude error and sloshing disturbance estimate given by dual observer as inputs. If the attitude error and disturbance exceeds some limit condition, then the controller should be switched to the alternative one.

4.1. Quaternion Partition Control. The quaternion partition control is an attitude control design method aiming for fast and stable attitude maneuver [15]. The method used in the LAM has three domains as shown in Figure 7: angle control, constrained angular rate, and nominal angular rate. In the angle control domain, a typical PID controller is implemented. In the nominal angular rate domain, a nominal constant angular rate is expected:

$$
\omega_{d}=d \theta_{r} \mathbf{q}_{e}
$$

where $d \theta_{r}$ is the nominal angular rate, $\mathbf{q}_{e}$ is the error quaternion vector, and $\omega_{d} \in \mathbb{R}^{3}$ is the target angular velocity. In the constrained angular rate domain, where the angular rate exceeds $d \theta_{m}$, constant thrust is adopted to reduce the angular rate quickly.

4.2. Phase and Gain Stabilization Filter. A light fuel tank without membrane is adopted in the LAM, which is different from Chang'E-3 or Chang'E-4 lander, and fuel sloshing should be considered in the control design to avoid instability. A pendulum model is used as the equivalent model for sloshing analysis. To improve phase and gain margin, sloshing filter is designed using the transfer function method and Nichols chart [14]:

$$
F(s)=\frac{a_{11} s^{2}+a_{12} s+1}{a_{13} s^{2}+a_{14} s+1} \frac{a_{21} s^{2}+a_{22} s+1}{a_{23} s^{2}+a_{24} s+1},
$$

where $a_{i j}, i=1,2, j=1,2,3,4$ are filter coefficients.

The nonlinear part like pulse-width-modulation (PWM) should also be considered in the design. A typical design case is considered that the filled volume reaches $45 \%$ with the control delay as $96 \mathrm{~ms}$. The Nichols charts of pitch, yaw, and roll axis of the LAM control system are shown in Figure 8 . As can be seen, satisfying phase and gain margins are obtained with a designed filter. The gain margin is larger than $6 \mathrm{~dB}$, and the phase margin is larger than $15 \mathrm{deg}$ during the descent and landing phase. Moreover, the linear part is 


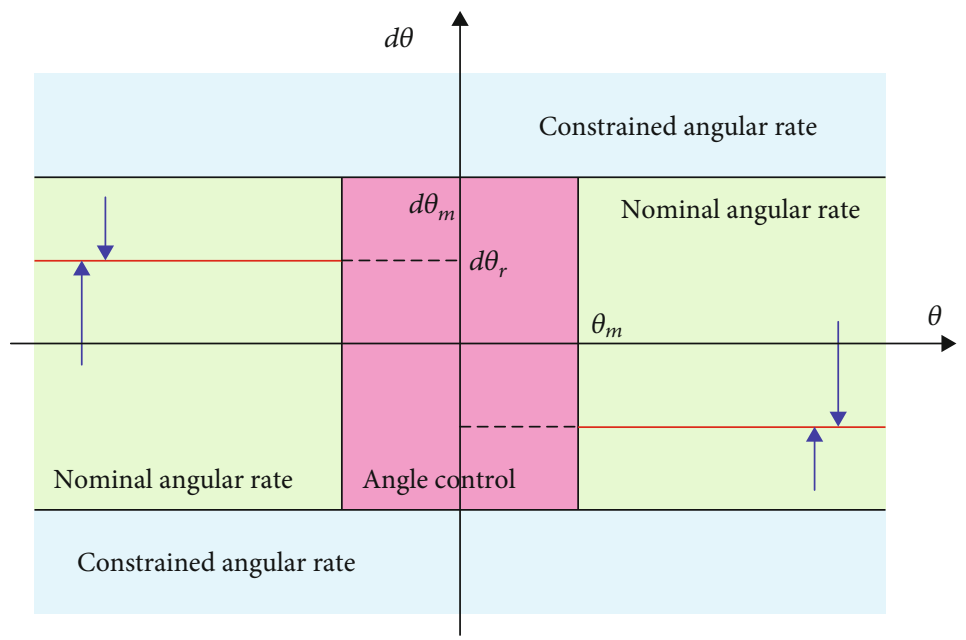

FIgURE 7: Domain definition of quaternion partition control.

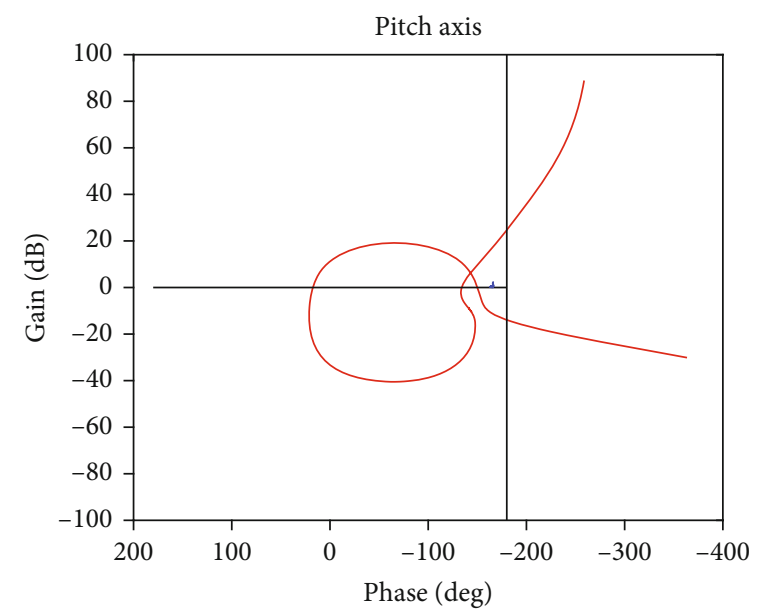

(a)

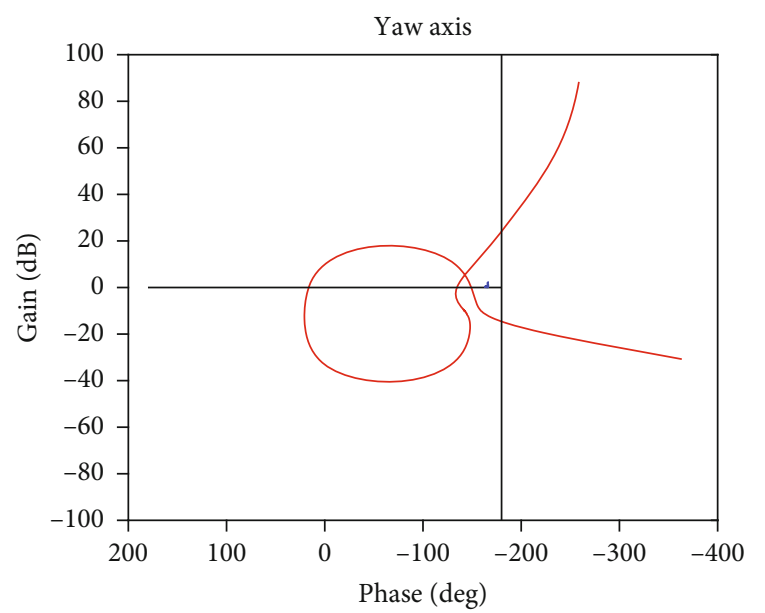

(b)

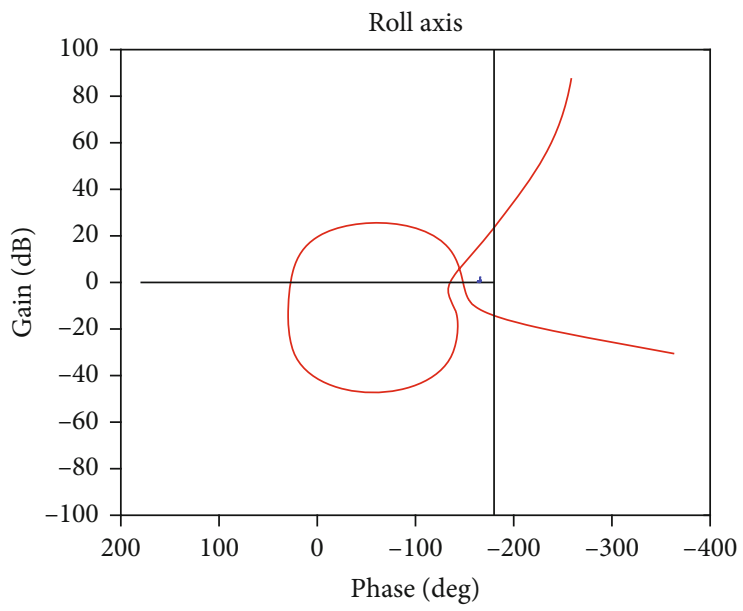

(c)

FIgUre 8: Nichols chart of the LAM with sloshing and PWM. 
far from the nonlinear part which is quite small in the Nichols chart.

4.3. Dual Observer. While phase and gain stabilization filter improves the robustness of the control system in the presence of liquid sloshing, disturbance estimate and compensation could achieve better control performance. A dual observer is designed with the structure as follows:

$$
\begin{aligned}
\widehat{\omega}_{k+1}^{-} & =\widehat{\omega}_{k}^{+}+J^{-1}\left(\tau_{k}+\widehat{\mathbf{d}}_{k}-\omega_{k+1} \times J \omega_{k+1}\right) \Delta t, \\
\widehat{\omega}_{k+1}^{+} & =\widehat{\omega}_{k+1}^{-}+L_{\omega}\left(\omega_{k+1}-\widehat{\omega}_{k+1}^{-}\right), \\
\widehat{\mathbf{d}}_{k+1} & =\widehat{\mathbf{d}}_{k}+L_{d} J\left(\omega_{k+1}-\widehat{\omega}_{k+1}^{-}\right),
\end{aligned}
$$

where $\omega \in \mathbb{R}^{3}$ is the measured angular velocity, $\widehat{\omega} \in \mathbb{R}^{3}$ is the estimated angular velocity, $J$ is the moment of inertia of the LAM, $\tau$ is the control torque, $\Delta t$ is the control sampling period, $\widehat{\mathbf{d}} \in \mathbb{R}^{3}$ is the estimated disturbance, and $L_{\omega}$ and $L_{d}$ are observer gain matrices.

The dual observer has two identical observers with same structure (Equation (15)) but different $L_{\omega}$ and $L_{d}$. Thus, the observer with a large gain is able to estimate more disturbances including fast-varying ones like sloshing disturbance, while a small gain observer could only track slow-varying disturbances like misalignment of center of mass. The difference between the two observer estimates can be regarded the fastvarying disturbances.

To determine which observer estimate should be used, some statistical values are used as follows:

$$
\begin{aligned}
E_{d m, i} & =\frac{\sum_{k=1}^{N} e_{d i}(k)}{N}, \\
E_{d \sigma, i} & =\sqrt{\frac{\sum_{k=1}^{N}\left(e_{d i}(k)-E_{d m}\right)^{2}}{N-1}},
\end{aligned}
$$

where $N$ is the accumulated number of points analyzed by the observer; $e_{d i}=\widehat{\mathbf{d}}_{a i}-\widehat{\mathbf{d}}_{b i}, \quad i=1,2,3$, is the difference between two estimated disturbances; and $E_{d m, i}$ and $E_{d \sigma, i}$, $i=1,2,3$, are the mean and standard deviation value of the difference. If the standard deviation of the fast observer is small enough, its output is selected as the disturbance estimate, otherwise the slow observer output is selected. It should be noted that the control torque command and reference angular velocity command should be synchronized in the observer. Some delay from previous control period should also be considered.

\section{Intelligent Heterogeneous Sensor Data Fusion}

The descent and landing navigation of the LAM makes use of multiple kinds of navigation sensors. The navigation system design of the LAM is based on Chang'E-3 lander [16]. Laser velocimeter and terrain camera are added to the LAM navigation system to increase system redundancy. The working range of each navigation sensor is shown in Figure 9. As can be seen, navigation results can be obtained from various kinds of navigation sensors. One of the most important problems in the navigation system is how to detect sensor fault and select the correct one. Intelligent heterogeneous sensor data fusion is implemented in the LAM navigation.

5.1. IMU Data Fusion. The LAM has one laser IMU and one fiber-optic IMU, and each has three gyroscopes and three accelerometers. In theory, three gyroscopes and accelerometers could generate enough data for the inertial navigation [17]. However, since laser gyroscopes and fiberoptic gyroscopes own different characteristics, it might result in low navigation precision if mixed gyroscopes are selected. To isolate sensor fault, each gyroscope and accelerometer output is compared with the other based on the mounting matrix. If any sensor output is different from the others, the other IMU is selected to be introduced into the navigation system. The comparison threshold could be dynamically adjusted according to the difference of the two IMU outputs.

The difference quaternion between two IMU gyroscopes is defined as [18]

$$
\mathbf{q}_{e}=\left[\begin{array}{cccc}
q_{4 a} & q_{3 a} & -q_{2 a} & -q_{1 a} \\
-q_{3 a} & q_{4 a} & q_{1 a} & -q_{2 a} \\
q_{2 a} & -q_{1 a} & q_{4 a} & -q_{3 a} \\
q_{1 a} & q_{2 a} & q_{3 a} & q_{4 a}
\end{array}\right]\left[\begin{array}{l}
q_{1 b} \\
q_{2 b} \\
q_{3 b} \\
q_{4 b}
\end{array}\right],
$$

where $\mathbf{q}_{a}=\left[q_{1 a}, q_{2 a}, q_{3 a}, q_{4 a}\right]^{T}$ and $\mathbf{q}_{b}=\left[q_{1 b}, q_{2 b}, q_{3 b}, q_{4 b}\right]^{T}$ are the integrated attitude quaternion from laser IMU and fiber-optic IMU, respectively. The attitude difference angle is defined as

$$
\theta_{I M U}=2 \arcsin \left\|\mathbf{q}_{e v}\right\|
$$

where $\mathbf{q}_{e v}$ is the vector part of $\mathbf{q}_{e}$. A smaller comparison threshold is preferred when $\theta_{\text {IMU }}$ is large, so that IMU fault could be isolated as soon as possible.

The difference velocity between two IMU accelerometers are compared in a similar way. If the difference velocity is large, then the comparison threshold becomes small.

5.2. Velocimeter Data Fusion. The radar and laser velocimeter could both provide the LAM velocity relative to the lunar surface, and each owns three beams. Similar to the IMU data fusion, velocimeter data fusion is also employed in the LAM navigation. Apart from empirical single beam data fault detection logic, like constant fault detection, out of measurement range fault detection, and so on, each radar and laser velocimeter beam data is compared with the other as well. Let $\mathbf{u}_{i} \in \mathbb{R}^{3}$ denote the $i$ th beam direction in the body frame, $i=1, \cdots, 6$, and $\delta v_{i}$ denote the difference between the beam measurement value and navigation value 


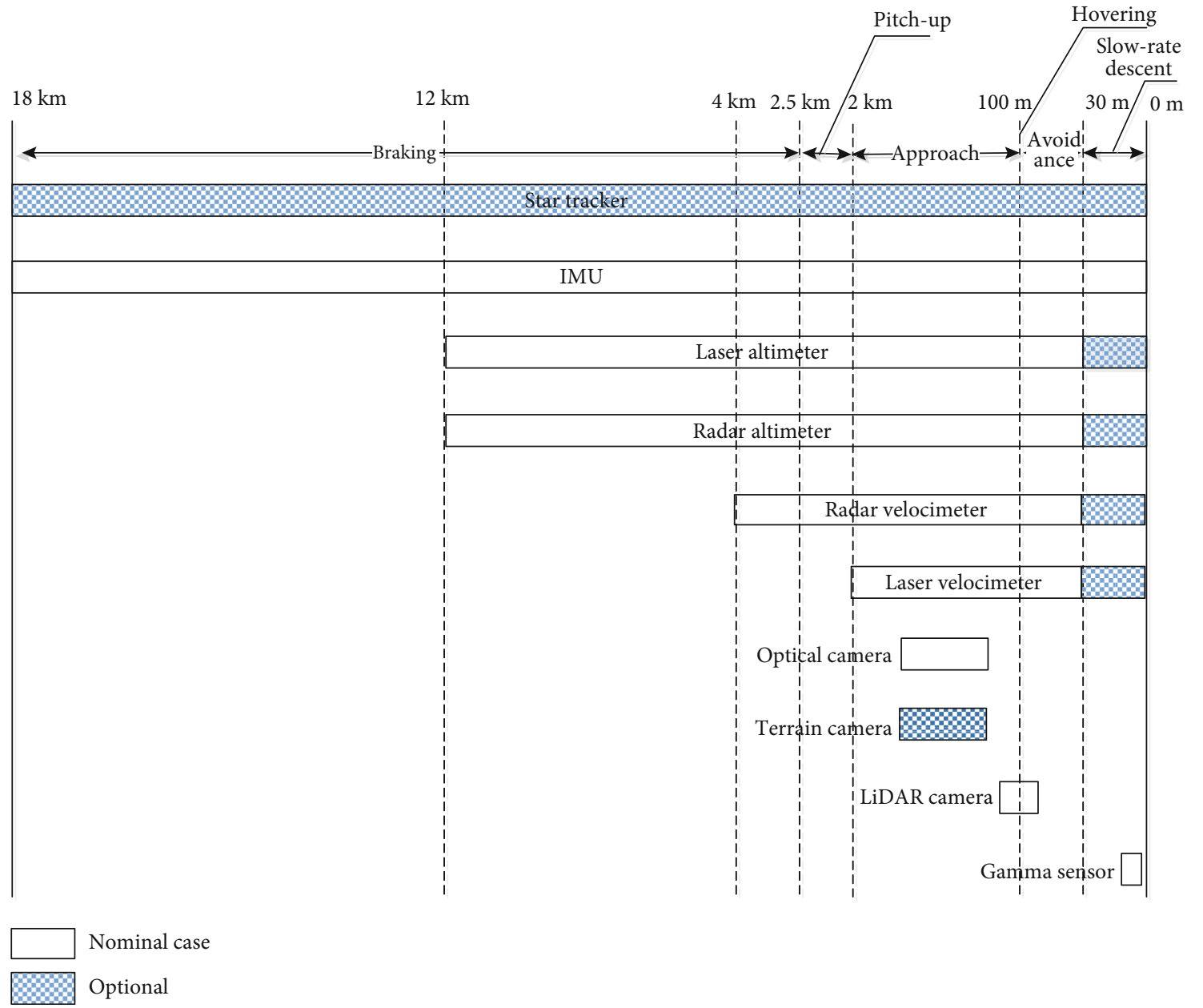

FIGURE 9: Working range of navigation sensors of the LAM.

along $\mathbf{u}_{i}$. Let

$$
C_{i j k l}=\mathbf{u}_{l}^{T}\left[\begin{array}{c}
\mathbf{u}_{i}^{T} \\
\mathbf{u}_{j}^{T} \\
\mathbf{u}_{k}^{T}
\end{array}\right]^{-1},
$$

where $1 \leq i<j<k<l \leq 6$. Then, the comparison error among these four beam measurement data can be calculated as

$$
\varepsilon_{i j k l}=\left|C_{i j k l}\left[\begin{array}{c}
\delta v_{i} \\
\delta v_{j} \\
\delta v_{k}
\end{array}\right]-\delta v_{l}\right| .
$$

If $\varepsilon_{i j k l}$ is smaller than some threshold, the grade of each of these four beams is increased, or otherwise each grade gets decreased. The comparison error for each permutation of these six beams is computed to select the three valid beam measurement data to be used in the navigation. If the measurement of radar velocimeter is quite different from that of laser velocimeter, then the radar data is preferred because the radar velocimeter has been successfully implemented by the Chang'E-3 and Chang'E4 landers.

\section{Flight Results}

On 1 Dec., 2020 22:57:17 (BJT), the main throttleable engine began ignition and the powered descent of the LAM started. The initial mass of the LAM was $3735.4 \mathrm{~kg}$, the altitude was about $18 \mathrm{~km}$, and the velocity was about $1.7 \mathrm{~km} / \mathrm{s}$. The flight results for the descent and landing are shown in Figures 10-15. The whole powered descent and landing phase duration, from the ignition of the $7500 \mathrm{~N}$ engine to the shutdown, was about 830 s.

In Figure 10, the altitude and velocity results are shown. The altitude went higher at the beginning of the trajectory since the powered descent began at the perilune point. The nominal altitude at the entry of the approach phase is designed as $2000 \mathrm{~m}$, while the actual flight altitude at the entry point is $2048 \mathrm{~m}$, which is quite close to the nominal one. The velocity was continuously decreasing during the descent phase. The thrust of the throttleable engine is shown 


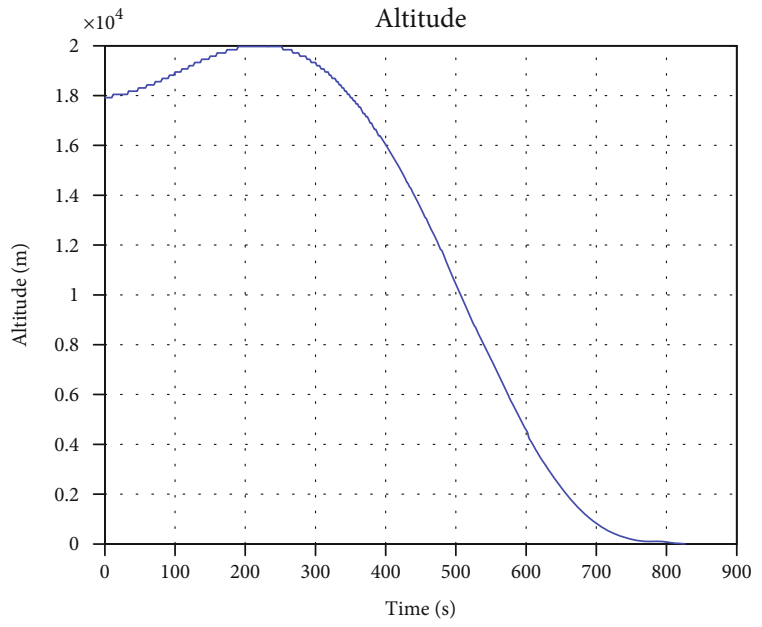

(a)
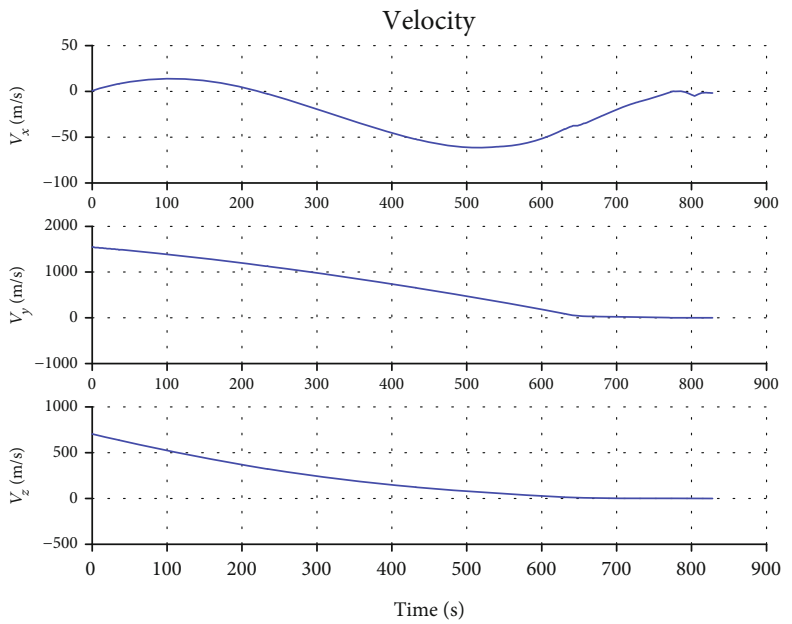

(b)

FIgURE 10: The LAM altitude and velocity relative to the lunar surface.

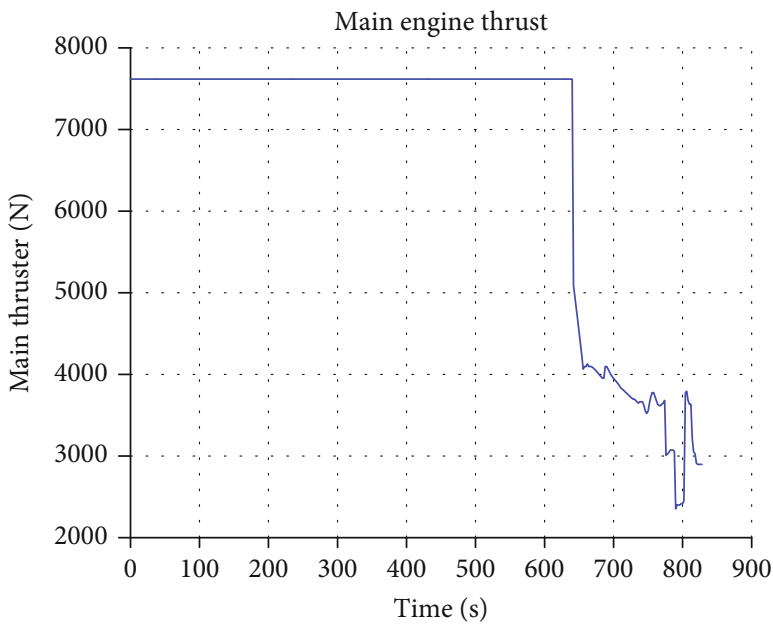

(a)

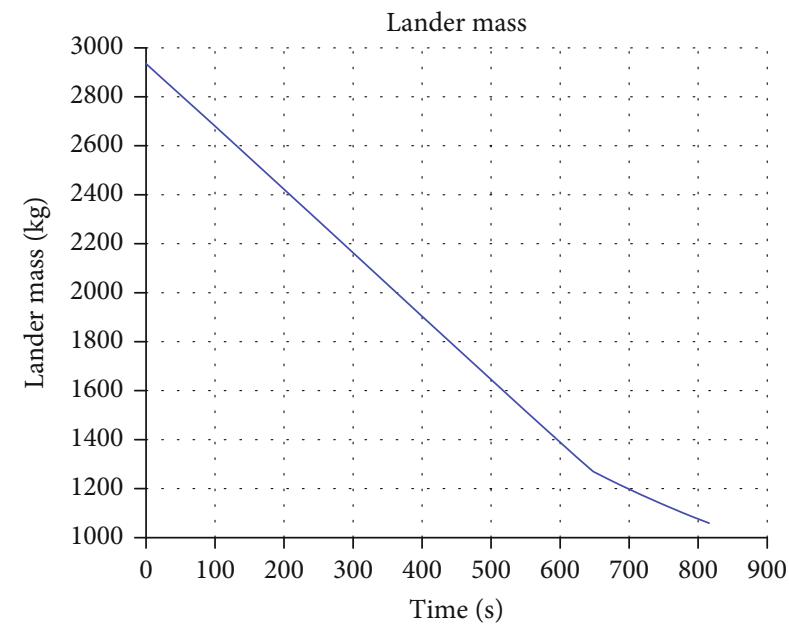

(b)

FIGURE 11: The LAM main engine thrust and lander mass.

in Figure 11(a). In the braking phase, which lasted about $640 \mathrm{~s}$, a constant maximum thrust was adopted and varying thrust was generated in the following phases according to the guidance command. Figure 11(b) shows the mass estimate history of the lander. The lander mass decreased with $150 \mathrm{~N}$ thrusters and $7500 \mathrm{~N}$ engine firing during the descent and landing phase. Since the $10 \mathrm{~N}$ thrusters also provided attitude control during descent, the ascender mass had decreased by $0.2 \mathrm{~kg}$. The attitude angles of the LAM mechanical frame relative to the local up-east-north frame and angular rates relative to the moon J2000 inertial frame are presented in Figure 12. A small angular velocity was seen in the braking phase due to guidance command, and then, the largest angular rates were experienced during the pitch-up phase. In the hovering phase, the attitude was reoriented as well.

The radar and laser sensor data measurements are shown in Figure 13. And their measurement residuals, differences between sensor measurements and navigation filter output, are shown in Figure 14. The sensor working range is shown in Figure 9. The laser altimeter could first provide valid altitude measurement since it has the farthest working range. When the altitude reached $12 \mathrm{~km}$, the radar altimeter could also provide valid measurement. The radar velocimeter was introduced to the navigation when the altitude was below $4 \mathrm{~km}$, and the laser velocimeter was used after the altitude reached $2 \mathrm{~km}$. The measurement results in Figure 13 and residuals in Figure 14 show that the radar and laser altimeters 

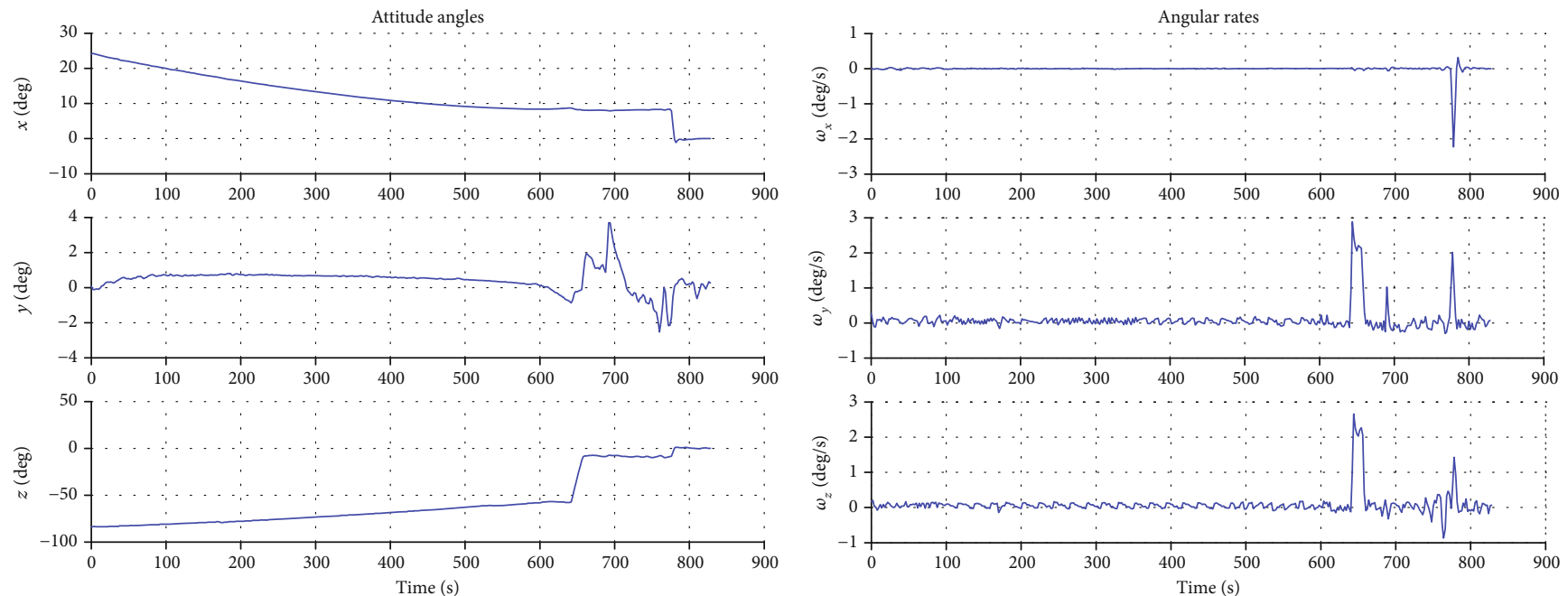

(a)

(b)

FIGURE 12: The LAM attitude angles and angular rates.
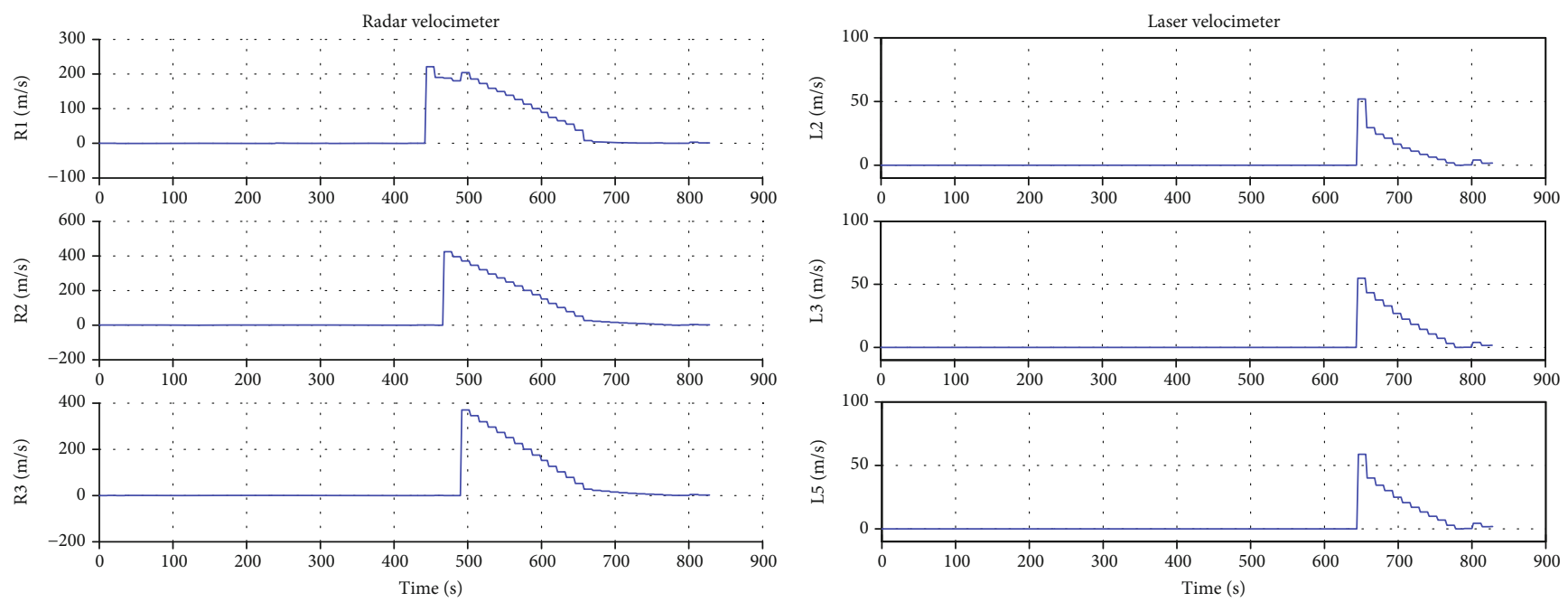

(a)

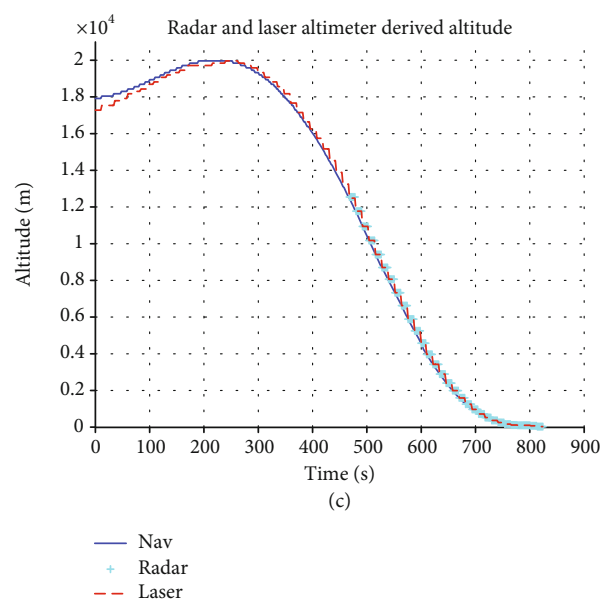

FIgURE 13: The radar and laser altimeter and velocimeter measurement. 

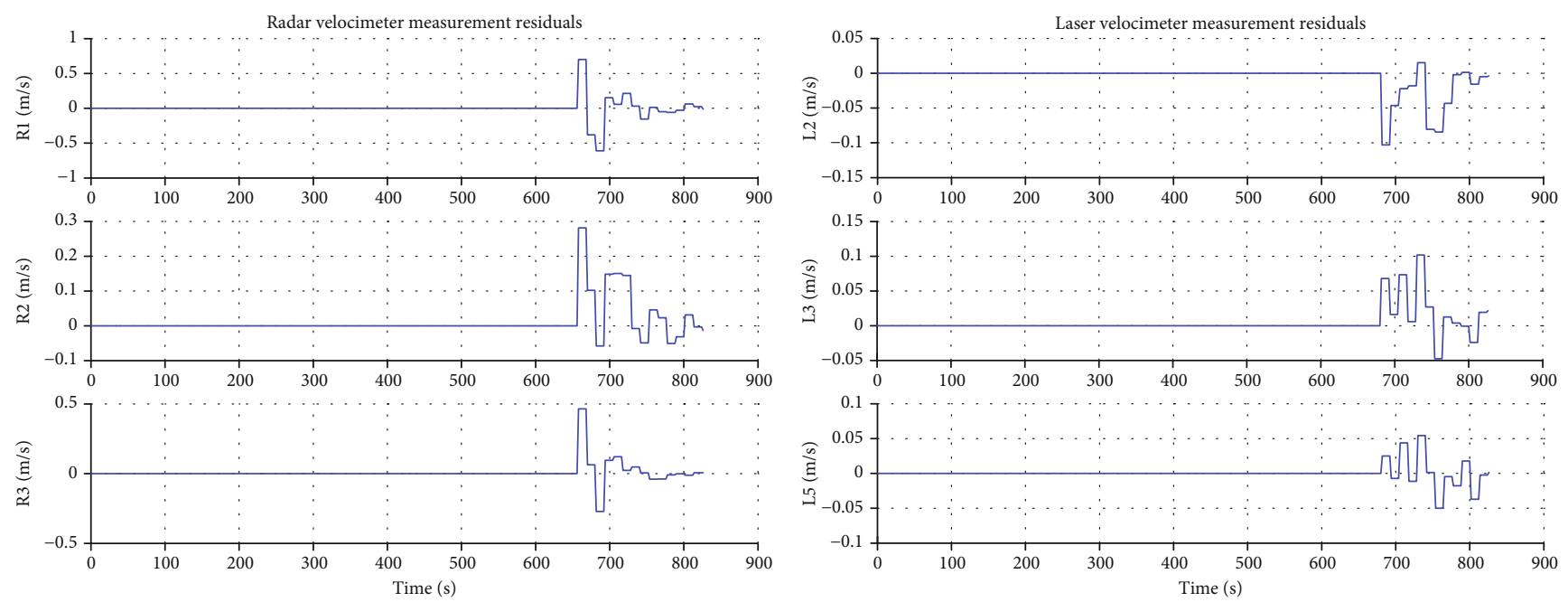

(a)

(b)
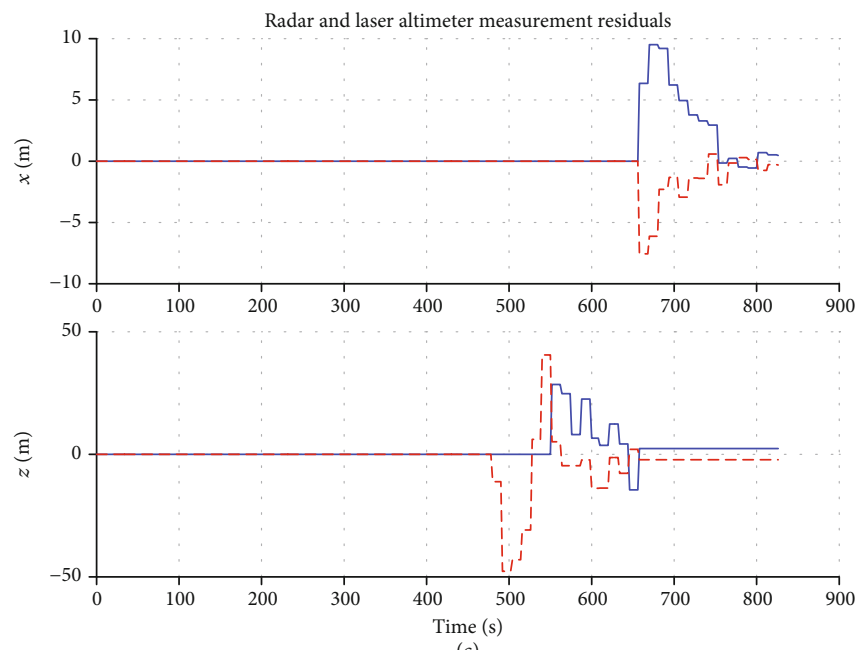

(c)

$$
\begin{aligned}
& \text { — Radar } \\
& \text { - } \text { Laser }
\end{aligned}
$$

FIgURE 14: The radar and laser altimeter and velocimeter measurement residuals.
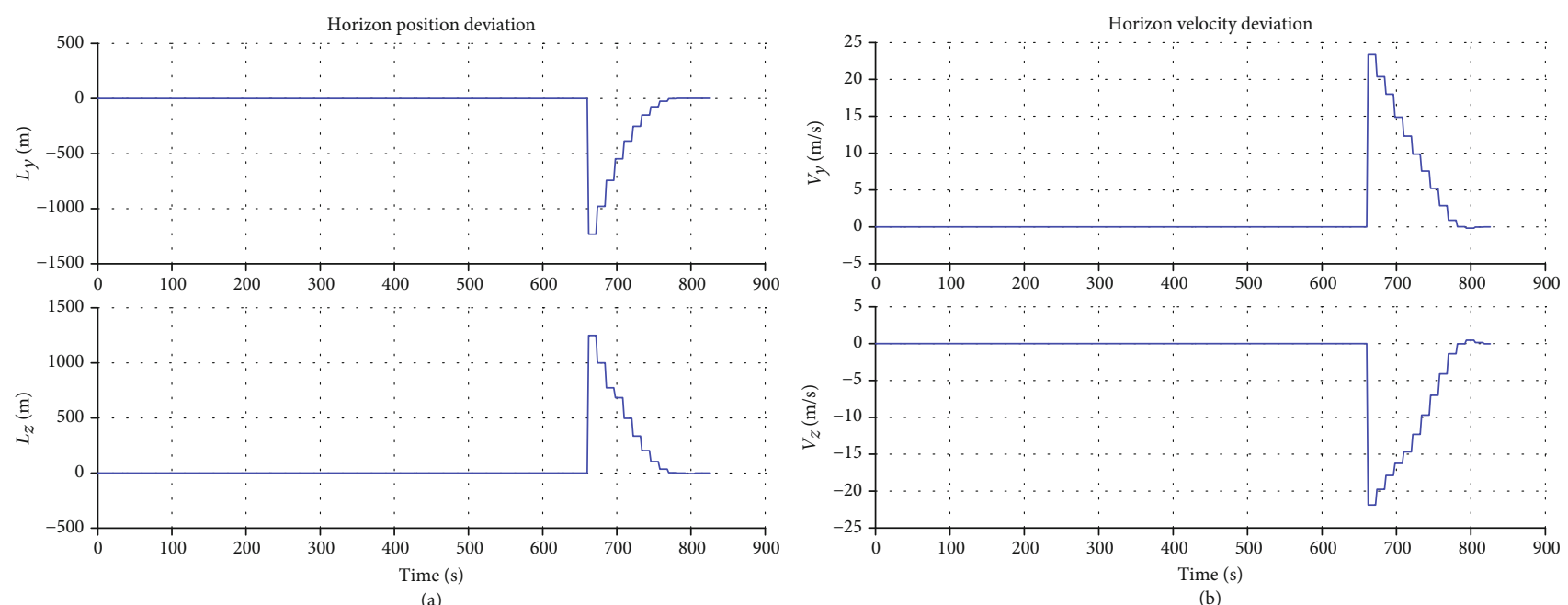

(a)

Figure 15: Hazard avoidance. 


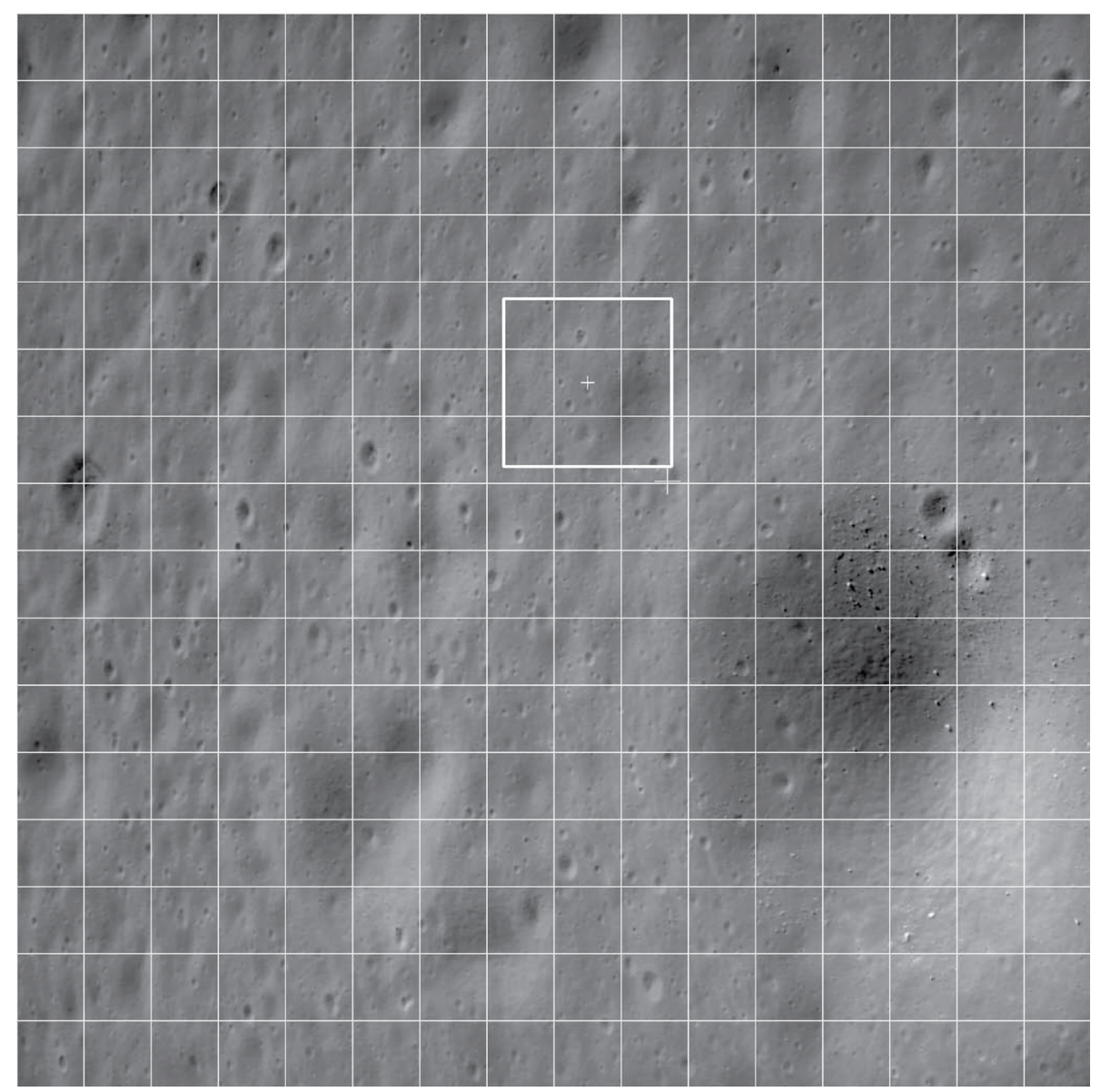

Figure 16: Optical image and coarse safe site.

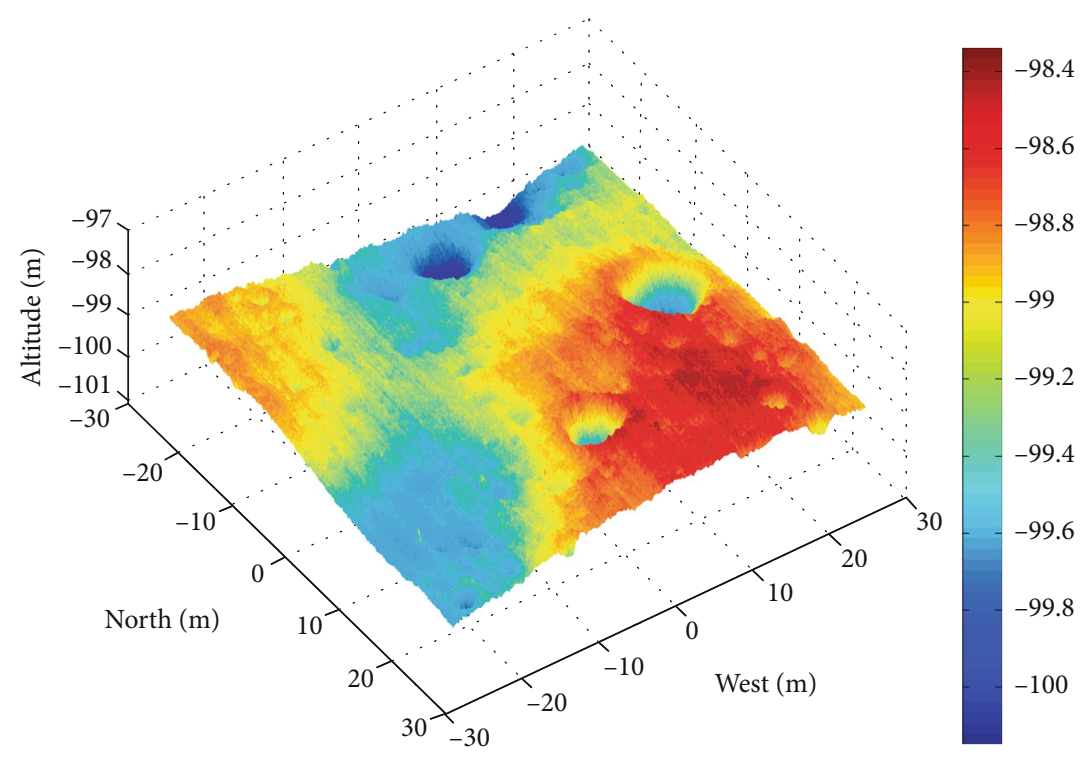

FIgURE 17: LiDAR image. 


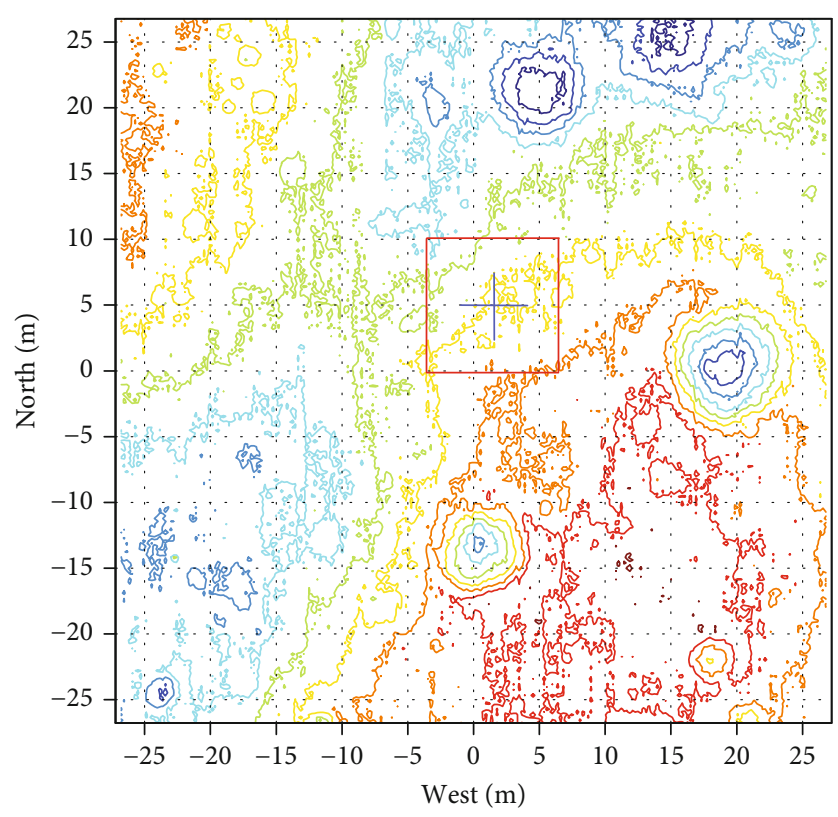

Figure 18: Fine safe site from LiDAR image.

TABle 2: Chang'E-5 LAM GNC flight results for descent and landing.

\begin{tabular}{lc}
\hline States & Results \\
\hline Landing horizontal velocity $(\mathrm{m} / \mathrm{s})$ & 0.0085 \\
Landing vertical velocity $(\mathrm{m} / \mathrm{s})$ & 1.99 \\
Landing angular rate $\left({ }^{\circ} / \mathrm{s}\right)$ & 0.03 \\
Angle between $+X$ axis and local up direction $\left({ }^{\circ}\right)$ & 0.2 \\
Angle between $-Y$ axis and local south direction $\left(^{\circ}\right)$ & 0.02 \\
Propellant mass used during descent $(\mathrm{kg})$ & 1898 \\
\hline
\end{tabular}

and velocimeters generally performed well on board since their residuals all converged to some small range as the LAM descended.

The hazard detection and avoidance results are shown in Figure 15. The horizonal position and velocity deviations converge to zero indicating that the target safe landing sites are achieved. The optical image and coarse safe landing site obtained from the optical camera during the approach phase, and the selected coarse safe landing site is located at the center of the box of the image as shown in Figure 16. The LiDAR image from the LiDAR camera is shown in in Figure 17 during the hovering phase. The new fine safe landing site is also located at the center of the box in Figure 18. These results show that the coarse and fine hazard detection cameras, algorithms, and avoidance maneuver all operate well as designed.

The key descent and landing LAM GNC flight results are given in Table 2 . The velocity at touchdown was estimated as about $2 \mathrm{~m} / \mathrm{s}$, the angular rate about $0.026 \%$, and the attitude error about $0.18^{\circ}$. The final actual landing site was $43.0617^{\circ} \mathrm{N}$ and $51.9161^{\circ} \mathrm{W}$.

\section{Conclusion}

The successful powered descent and landing of the Chang'E-5 LAM demonstrates the effectiveness of the GNC design. Navigation sensors all performed as expected even though some of them were first implemented in space. The intelligent guidance and other guidance approaches guaranteed the success of the soft landing, coarse hazard avoidance, fine hazard avoidance, and other activities during descent phase. The attitude controller provides enough robustness for the system with sloshing effects and fast attitude maneuver in the presence of large attitude deviations. These techniques contribute to the success of the descent and landing the LAM.

\section{Data Availability}

The data used to support the findings of this study are available from the author upon reasonable request.

\section{Conflicts of Interest}

The authors declare that they have no conflicts of interest.

\section{References}

[1] P. Ye and J. Peng, "Deep space exploration and its prospect in China," Engineering Science, vol. 8, pp. 13-18, 2006.

[2] F. V. Bennett, Apollo experience report - mission planning for lunar module descent and ascent, Tech. Rep, National Aeronautics and Space Administration, Washington D. C, 1972.

[3] J. L. Goodman, "Apollo 13 guidance, navigation, and control challenges," in AIAA SPACE 2009 Conference \& Exposition, Pasadena, California, 2009.

[4] S. Dutta and R. D. Braun, "Statistical entry, descent, and landing performance reconstruction of the Mars science 
laboratory," Journal of Guidance, Control, and Dynamics, vol. 51, pp. 1048-1061, 2014.

[5] H. Zhang, J. Liang, X. Huang et al., "Autonomous hazard avoidance control for Chang'E-3 soft landing," Scientia Sinica Technologica, vol. 44, pp. 559-568, 2014.

[6] H. Zhang, Y. Guan, M. Cheng et al., "Guidance navigation and control for Chang'E-4 lander," Scientia Sinica Technologica, vol. 49, pp. 1418-1428, 2019.

[7] H. Yin, "Development history of China's moon probe project," Tianjin Science \& Technology, vol. 44, pp. 79-87, 2017.

[8] A. E. Bryson and Y.-C. Ho, Applied Optimal Control, Blaisdell Publishing Company, Waltham, Mass, 1969.

[9] R. L. McHenry, T. J. Brand, A. D. Long, B. F. Cockrell, and J. R. Thibodeau III, "Space shuttle ascent guidance, navigation, and control," The Journal of the Astronautical Sciences, vol. 27, pp. 1-38, 1979.

[10] J. R. Rea and R. H. Bishop, "Analytical dimensional reduction of a fuel optimal powered descent subproblem," in AIAA Guidance, Navigation, and Control Conference, Toronto, Ontario Canada, 2010.

[11] A. R. Klumpp, “Система ведения спуска лунного модуля Аполло," Automatica, vol. 10, no. 2, pp. 133-146, 1974.

[12] B. A. Steinfeldt, M. J. Grant, D. M. Matz, and R. D. Braun, "Guidance, navigation, and control technology system trades for mars pinpoint landing," in AIAA Atmospheric Flight Mechanics Conference and Exhibit, Honolulu, Hawaii, 2010.

[13] E. C. Wong and G. Singh, "Guidance and control design for hazard avoidance and safe landing on Mars," Journal of Guidance, Control, and Dynamics, vol. 43, pp. 378-384, 2006.

[14] B. Wie, Space Vehicle Dynamics and Control, American Institute of Aeronautics and Astronautics, Reston, Virginia, 2nd edition, 2006.

[15] H. Zhang, Y. Guan, J. Hu, and Z. Wang, "A novel attitude control strategy based on quaternion partition," Acta Automatica Sinica, vol. 41, no. 7, pp. 1341-1349, 2015.

[16] H. Zhang, J. Li, Y. Guan, and X. Huang, "Autonomous navigation for powered descent phase of Chang'E-3 lunar lander," Control Theory \& Applications, vol. 31, pp. 1686-1694, 2014.

[17] P. G. Savage, "A unified mathematical framework for strapdown algorithm design," Journal of Guidance, Control, and Dynamics, vol. 29, no. 2, pp. 237-249, 2006.

[18] B. Wie, H. Weiss, and A. Arapostathis, "Quarternion feedback regulator for spacecraft eigenaxis rotations," Journal of Guidance, Control, and Dynamics, vol. 12, no. 3, pp. 375-380, 1989. 